\title{
Diffuse degeneration of cerebral white matter resembling so-called Binswanger's disease and symmetrical necrosis of the globus pallidus associated with acute porphyria and cerebral atherosclerosis
}

\author{
W. THOMAS SMITH AND S. R. F. WHITTAKER \\ From the Department of Pathology, University of Birmingham, and Warwick Hospital
}

SYNOPSIS A 59-year-old woman admitted to hospital in coma also showed generalized muscular rigidity and porphyria. After two days she became fully conscious but the muscular rigidity increased and persisted until death occurred 12 weeks later.

The brain showed diffuse degeneration of the white matter; symmetrical necrosis of the globus pallidus was associated with severe localized atherosclerosis.

The lesions probably resulted from the combined effects of porphyria and atherosclerosis. It is suggested that in later life unusual cerebral lesions may occur with porphyria because of associated atherosclerosis. The porphyria may be overlooked clinically.

\section{CASE REPORT}

A spinster of 59 who had previously been in good health complained of feeling drowsy one evening and was found unconscious in bed the next morning. She was admitted to hospital in coma with striking rigidity affecting the muscles of the neck, both arms, and both legs. Deep reflexes were increased. Plantar reflexes were extensor. Blood pressure was $125 / 80 \mathrm{~mm}$. $\mathrm{Hg}$. Lumbar puncture showed clear fluid under normal pressure containing less than 2 cells per $\mathrm{ml}$. and protein $30 \mathrm{mg}$. $/ 100 \mathrm{ml}$. The urine was very dark, containing large quantities of porphobilinogen and porphyrins when examined by the methods described by Whittaker and Whitehead (1956). Liver function tests were normal. Barbiturates were not detected in the blood. The blood urea level was $66 \mathrm{mg}$. $/ 100 \mathrm{ml}$. The Wassermann reaction was negative.

During the next two days the patient became fully conscious but the rigidity in her arms became even more marked and it was impossible to straighten them at the elbow joints. Rigidity also developed in the facial muscles and tube feeding was necessary. An electroencephalogram showed generalized irregular slow waves completely replacing any basic rhythmic activity. A muscle biopsy (taken from the right deltoid by Dr. A. L. Woolf) showed definite, though slight, involvement of the lower motor neurone.

Received for publication 23 September 1962
The patient remained in this curious state of rigidity and akinesia, but eventually developed a urinary tract infection and bedsores and she died from bronchopneumonia 12 weeks after the onset of the illness. During the whole period she passed porphobilinogen in the urine but the amount became substantially less after the first four weeks. Her mind remained clear, she was able to speak, and she gave no history of any previous illness or family history suggestive of acute porphyria.

\section{NECROPSY FINDINGS}

Coronal sections of the brain after fixation showed slight symmetrical dilatation of the lateral ventricles, and small areas of necrosis in the medial parts of both pallida, which on the right side also involved the adjacent part of the internal capsule. There was mild atheroma of the basal arteries.

The spinal cord and the peripheral nerves and muscles showed nothing of note. The lungs showed patchy bronchopneumonia. No other relevant abnormalities were seen.

\section{HISTOLOGY}

CENTRAL NERVOUS SYSTEM Sections from blocks doubly embedded in celloidin-paraffin wax and also 


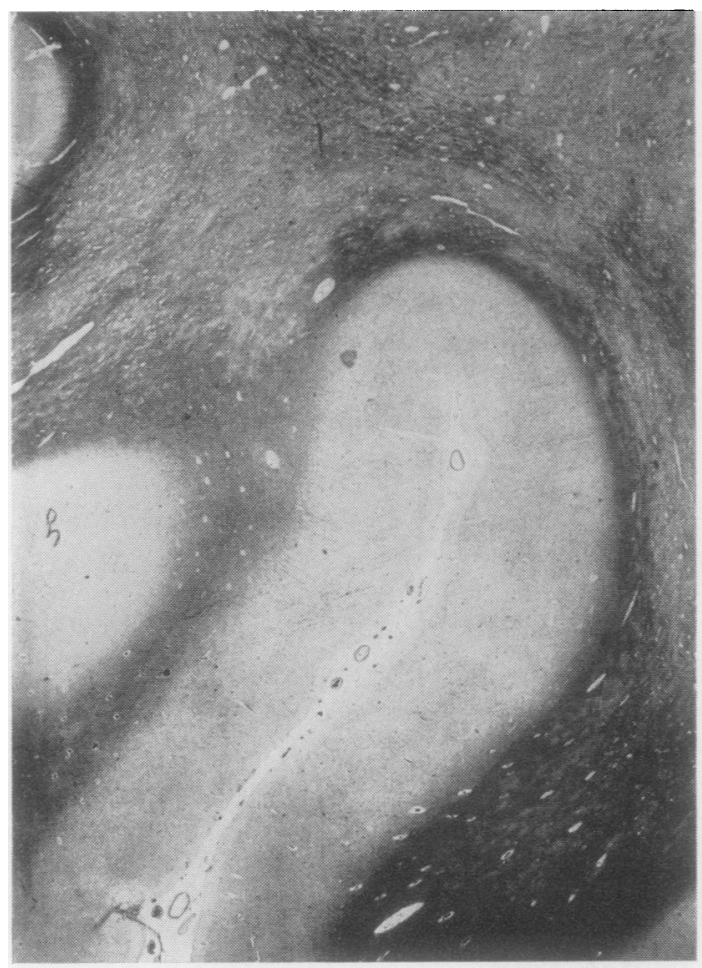

FIG. 1. Pre-Rolandic region. Diffuse, irregular demyelination. Subcortical arcuate fibres relatively spared. Weil's myelin stain. $\times 4 \frac{1}{2}$.

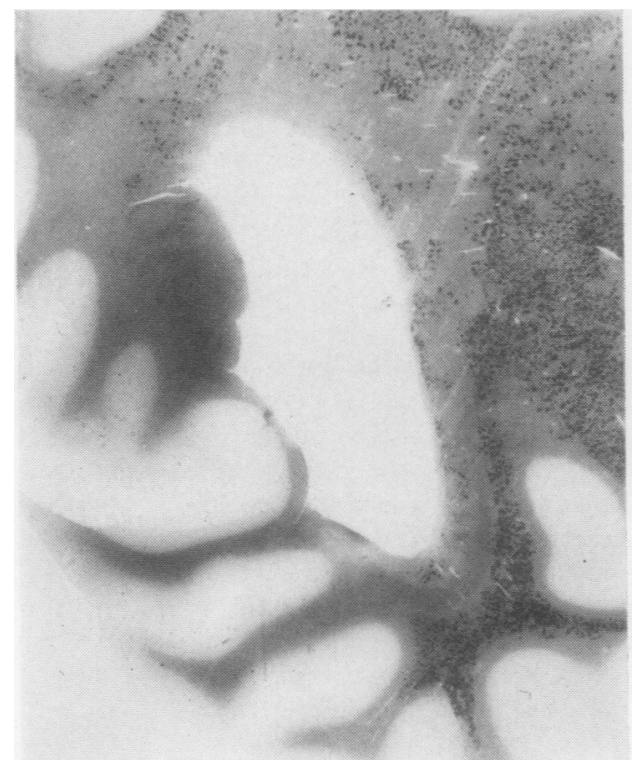

FIG. 2. Posterior horn of lateral ventricle. Diffuse degeneration of surrounding white matter. Frozen section. Marchi. $\times 2$ approximately. frozen sections were examined with routine general and neurohistological stains.

Throughout the white matter of both cerebral hemispheres myelin stains show numerous ill-defined, pale areas of degeneration, which vary in size from 0.5 to $10 \mathrm{~mm}$. in cross section, and in many places coalesce to involve much larger areas (Fig. 1). In a few of the smaller areas there is complete demyelination, although variable numbers of swollen and fragmented myelin sheaths are usually seen, especially at the periphery of the lesions. The myelin of the optic nerves, cerebellum, pons, medulla, and spinal cord appears normal. A consistent feature is an irregular, narrow band of normally stained myelin immediately beneath the cerebral cortex (Figs. 1 and 2). Higher magnification of the white matter between the degenerated areas shows swelling and beading of the myelin sheaths but no definite reduction in their number.

In frozen sections the areas of degeneration contain abundant, rounded, granular masses of lipid material which is sudanophilic and Marchi positive (Fig. 2) and P.A.S. negative. Some of these lipid masses represent bloated microglial phagocytes although many appear to be extracellular. The majority are unstained in embedded sections and appear as clear spaces, sometimes related to a small round or elongated nucleus (Fig. 3). There is diffuse stippling with free-lying sudanophilic particles, both in the degenerated areas and in the intervening white matter, where occasional rounded lipophages are also noted. Short lengths of beaded, myelinated fibres, which are sudanophilic and Marchi positive, are seen in the areas of degeneration, where there is also swelling, fragmentation, and reduction in the number of axons. The axonal degeneration is quantitatively less severe than the myelin degeneration.

There is astrocytic hypertrophy throughout the white matter, especially within or adjacent to the degenerating zones (Fig. 3). Glial fibres are scanty except in the small foci of complete demyelination and giant multinucleated astrocytes are not seen. The oligodendrocytes show cytoplasmic swelling and are reduced in number; in some areas of degeneration they have completely disappeared. There are no $\mathcal{N}$ inclusion bodies. The perivascular spaces are $N$ frequently dilated and contain eosinophilic fluid and $\mathcal{N}$ variable numbers of lymphocytes and phagocytes.

In the grey matter of the hemispheres there is

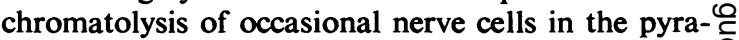
midal layers of the cerebral cortex and hippocampus, and in the cranial nerve nuclei. Other nerve cells are shrunken and chromophilic but vacuolation is not ${ }^{-}$ seen. In the cerebral cortex small groups of neurones $\overrightarrow{\mathbb{D}}$ in the middle layers stain poorly, and in the inner $\frac{?}{\mathbb{D}}$ layer there is patchy loss of nerve cells, perineuronal 


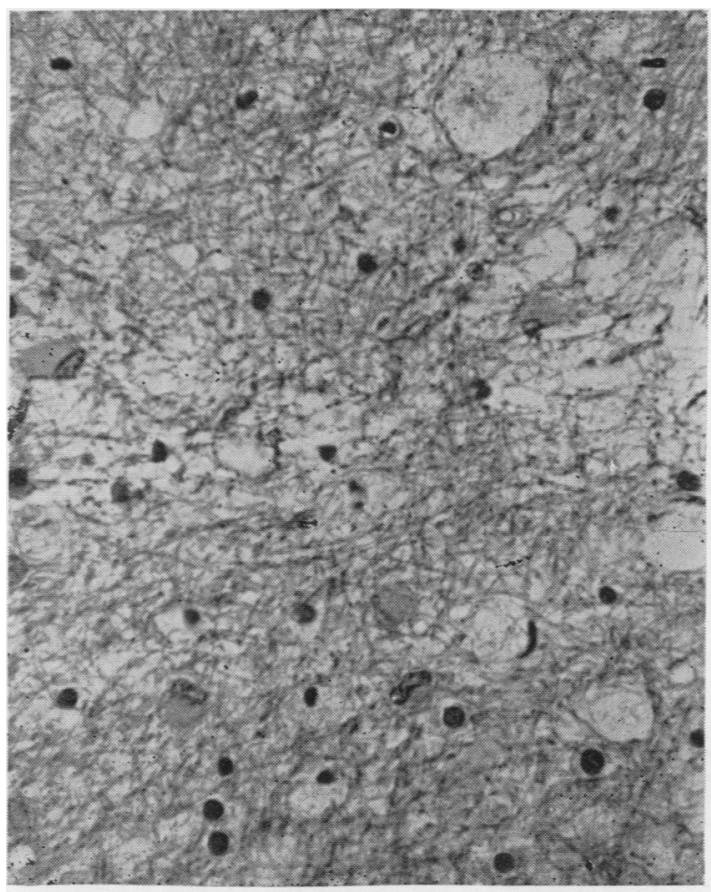

FIG. 3. Occipital region. Clear spaces containing granular material indicate sites of lipid masses. Astrocytic hypertrophy. Phosphotungstic acid-haematoxylin. $\times 620$.

satellitosis, and astrocytic hypertrophy. Both thalami show similar but more extensive neuronal degeneration, maximal in the lateral nuclei. Many neurones throughout the brain contain an excess of lipofuscin pigment. In the cerebellum random Purkinje cells and nerve cells in the dentate nuclei show either chromatolysis or atrophy and homogeneous staining, together with related astrocytic hypertrophy. The anterior horn cells in the spinal cord show marked central chromatolysis.

Both pallida show clearly defined areas of necrosis involving their dorso-medial segments and the adjacent margins of the internal capsules. On both sides the lesions are bordered by a thin zone of hypertrophied astrocytes, amongst which there is a meshwork of glial fibres. Within the lesions are irregular masses and strands of necrotic debris, separating and also infiltrated by foamy and granular phagocytes. Small arteries traversing the necrotic areas show perivascular fibrosis, pronounced medial fibrosis, calcification and siderosis, and fibro-fatty intimal proliferation resulting in narrowing and in places obliteration of their lumina. There is evidence of recanalization in several occluded vessels. Elsewhere in the lentiform nuclei the small arteries show much less severe degeneration and their perivascular spaces are dilated. Within the rest of the brain the arterioles show fibrous thickening which is not exceptional for the age of the patient. There is adventitial and medial fibrosis of the small arteries traversing the subarachnoid space. The main arteries of the circle of Willis show similar fibrosis, together with mild nonocclusive intimal atheroma and thickening of their internal elastic lamellae.

Peripheral nerves were not available for histological examination. Bronchopneumonia was confirmed. In the muscles and other organs there were no relevant changes.

\section{DISCUSSION}

Degeneration of the subcortical white matter associated with symmetrical pallidal necrosis has very occasionally followed on a period of coma resulting from anoxia due to inhalation of coal gas, carbon monoxide, nitrous oxide, or other noxious gases (Courville, 1957; Denny-Brown, 1962; Plum, Posner, and Hain, 1962). This mechanism did not account for the onset of coma in the present case, neither was there evidence of anoxia during the period of coma.

Although demyelination of the peripheral nerves is a well-known complication of acute porphyria, cerebral demyelination has been described in only a few cases and has then been focal in type and usually perivascular in distribution (Baker and Watson, 1945; Denny-Brown and Sciarra, 1945; Abbott and Evans, 1946; Gibson and Goldberg, 1956). According to Goldberg (1959) the most frequent age of onset of acute porphyria in women is in the third decade and in his series of 50 cases in only five (four women and one man) did it first occur over the age of 40 years. Because of this early age incidence it is unlikely that cerebral atherosclerosis was a significant factor in the pathogenesis of the cerebral lesions hitherto recorded in this condition. In the present case of late onset, however, it is likely that the widespread degeneration of cerebral white matter resulted from the combined effects of cerebral atherosclerosis and porphyria.

We were unable to find any precipitating factor for the attack of porphyria or any incident in the past medical history indicating a previous attack. As the patient recovered consciousness while still excreting large amounts of porphobilinogen, and as this only lessened after about four weeks, the initial coma cannot be entirely attributed to the porphyria. We have not found a previous record of a similar clinical picture in porphyria; the nearest example is case 3 of Schwarz and Moulton (1954), in which there was tonic contraction of shoulder, facial, and thigh adductor muscles. The absence of mental changes throughout our patient's illness was a surprising feature in view of the considerable interruption of 
cortical association and projection systems. Strich (1956) described spastic quadriparesis in patients showing diffuse cerebral demyelination after head injury but severe dementia was also present. Rigidity is a frequent finding with symmetrical pallidal necrosis due to asphyxic anoxia (Denny-Brown, 1962).

Focal necrotic cerebral lesions have been found before with porphyria and attributed to vasopasm (Hierons, 1957), and Denny-Brown and Sciarra (1945) held that the clinical manifestations might result from vasoconstriction during phases of hypertension. Degeneration of the white matter resembling that found in our case has not previously been reported with porphyria. Similar degeneration occurs in the condition commonly known as Binswanger's disease, attributed to Binswanger (1894), and subsequently discussed by Alzheimer (1898), Farnell and Globus (1932), Davison (1942), Neumann (1947), and Olszewski (1962); the cause has been held to be 'arteriosclerosis' of the deep penetrating arteries supplying the white matter. The differential histological diagnosis includes diffuse sclerosis (Schilder's disease) and the recently described progressive multifocal leucoencephalopathy which is most commonly associated with malignant reticuloses (Richardson, 1961). In the case of Binswanger's disease reported by Farnell and Globus (1932) there was widespread muscular rigidity similar to that shown by our patient. Wertham and Wertham (1934) and Neumann (1947) suggested that the lesions of Binswanger's disease would be found more often if 'arteriosclerosis' alone was the cause. Our findings support the view that encephalopathy of the Binswanger type can result from many causes, including cerebral atherosclerosis, which may act alone or in various combinations. Olszewski (1962) suggested that obscure

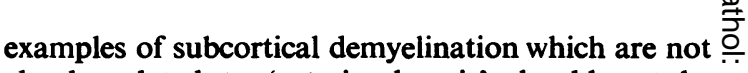
clearly related to 'arteriosclerosis' should not be $\vec{F}$ associated with the name of Binswanger, and our $\stackrel{\text { ? }}{+}$ case falls into this category.

From the practical point of view this case is $\frac{\overline{\bar{D}}}{\overline{\mathrm{D}}}$ instructive because it indicates that in later life $\frac{\pi}{\sigma}$ cerebral lesions complicating porphyria may be $\propto$ unusual in type due to associated atherosclerosis. ڤ The porphyria in such a case may not be noticed $\overrightarrow{0}$ clinically.

We are indebted to Mr. T. P. Whitehead, F.R.I.C., for the biochemical findings, Dr. A. L. Woolf for the report on the muscle biopsy, and Dr. A. P. Prior for the $\vec{a}$ necropsy findings and for sending on the brain and other or tissues for histological examination.

\section{REFERENCES}

Abbott, K. H., and Evans, H. S. (1946). Bull. Los. Angeles neurol. Soc., 11, 20.

Alzheimer, A. (1898). Mschr. Psychiat. Neurol., 3, 101.

Baker, A. B., and Watson, C. J. (1945). J. Neuropath., 4, 68.

Binswanger, O. (1894). Berl. klin. Wschr., 31, 1103, 1137, and 1180.

Courville, C. B. (1957). J. nerv. ment. Dis., 125, 534.

Davison, C. (1942). J. Neuropath., 1, 42.

Denny-Brown, D., and Sciarra, D. (1945). Brain, 68, 1.

(1962). The Basal Ganglia and their Relationship to Disorders of Movement, pp. 57-62, Oxford University press, London.

Farnell, F. J., and Globus, J. H. (1932). Arch. Neurol. Psychiat. W (Chicago), 27, 593.

Gibson, J. B., and Goldberg, A. (1956). J. Path. Bact., 71, 495.

Goldberg, A. (1959). Quart. J. Med., 28, 183.

Hierons, R. (1957). Brain, 80, 176.

Neumann, Meta, A., (1947). J. Geront., 2, 57.

Olszewski, J. (1962). Wld Neurol., 3, 359.

Plum, F., Posner, J. B., and Hain, R. F. (1962). Arch. intern. Med., $110,18$.

Richardson, E. P. Jr., (1961). New Engl. J. Med., 265, 815.

Schwarz, G. A., and Moulton, J. A. L. (1954). Arch. intern. Med., 94, 221.

Strich, Sabina, J. (1956). J. Neurol. Neurosurg. Psychiat., 19, 163.

Wertham, F., and Wertham, Florence (1934). The Brain as an Organ, p. 199. Macmillan, New York.

Whittaker S. R. F., and Whitehead, T. P. (1956). Lancet, 1, 547. 